EPJ Web of Conferences 41, 09018 (2013)

DOI: $10.1051 /$ epjconf/20134109018

C) Owned by the authors, published by EDP Sciences, 2013

\title{
Real-time observation of ultrafast Rabi oscillations between excitons and plasmons in metal/molecular aggregate hybrid nanostructures
}

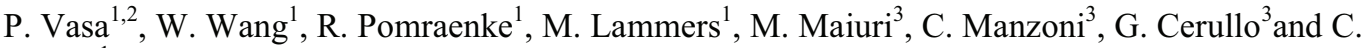 \\ Lienau $^{1}$ \\ ${ }^{1}$ Institut für Physik, Carl von Ossietzky Universität, 26111 Oldenburg, Germany \\ ${ }^{2}$ Department of Physics, Indian Institute of Technology Bombay, 400076 Mumbai, India \\ ${ }^{3}$ IFN-CNR, Dipartimento di Fisica, Politecnico di Milano, Milano, Italy
}

\begin{abstract}
We demonstrate ultrafast coherent manipulation of the normal mode splitting in metal/molecular-aggregate nanostructures by real-time observation of Rabi oscillations between excitons and surface-plasmon-polaritons. Oscillations in exciton density on a 10-fs timescale control the Rabi splitting.
\end{abstract}

\section{Introduction}

Hybrid nanostructures comprising semiconductors and metals currently attract substantial attention. In appropriately designed hybrids, the radiative coupling between the exciton dipole moment and local surface plasmon polariton (SPP) field can be so large that it greatly exceeds the linewidth of the uncoupled exciton and SPP resonances. In favorable cases, Rabi splittings of up to $700 \mathrm{meV}$ have
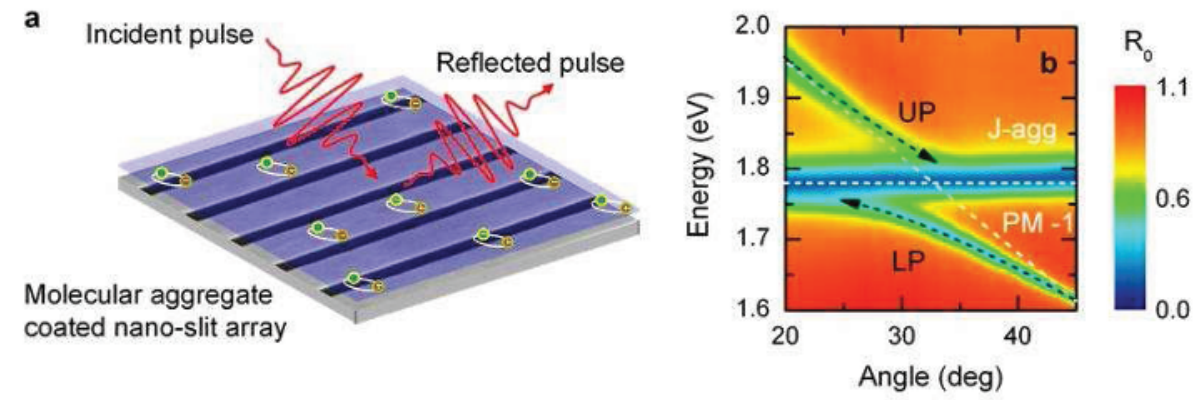

Figure 1: (a) Schematic of a metal/molecular hybrid nanostructure and (b) experimental linear reflectivity spectra of the metal/molecular aggregate hybrid nanostructures showing excitonSPP polariton formation with $\hbar \Omega_{N M S} \sim 110 \mathrm{meV}$.

been observed, almost as large as the bandgap of the semiconductor and hence approaching the socalled ultrastrong coupling regime [1-3]. This unique combination of SPP-like propagation, excitonic optical nonlinearities and ultrastrong radiative coupling means that such hybrid nanostructures form a new class of functional materials offering novel possibilities to efficiently localize, switch and

This is an Open Access article distributed under the terms of the Creative Commons Attribution License 2.0, which permits unrestricted use, distribution, and reproduction in any medium, provided the original work is properly cited. 
guide light on the nanometer length and femtosecond time scales [4]. So far, their optical properties have been studied by quasi-stationary and/or incoherent optical spectroscopy whereas any direct information on the coherent dynamics of radiatively coupled exciton-SPP excitations is lacking. Here, we present the first real-time observation of the exciton and plasmon dynamics in the strong coupling regime and demonstrate the coherent manipulation of the coupling energy by controlling the exciton density as well as the mixing of the wavefunctions.

\section{Metal/molecular hybrid nanostructure and its linear response}

We investigate a metal/molecular aggregate, hybrid nanostructure consisting of a $50 \mathrm{~nm}$ thick Jaggregated cyanine dye-polymer film spin-coated on a gold grating [Fig. 1a]. The exciton resonance in the aggregated dye film at room temperature occurs at $1.79 \mathrm{eV}$. The grating period of 400-460 nm is chosen so that the aggregate exciton is in resonance with the dye-metal SPP at an incidence angle of $\sim 30^{\circ}$. Strong, linear exciton-SPP coupling in these hybrid nanostructures is investigated by measuring the angle-resolved $p$-polarized reflectivity spectra shown in Fig. 1b. Spectra reveal significant bending of the SPP and exciton resonances due to the formation of the upper (UP) and lower (LP) SPP-exciton polaritons, [2,5] with normal mode splitting, $\Omega_{\mathrm{NMS}} \sim 110 \mathrm{meV}$. This strong normal mode splitting reflects the coupling of J-aggregate excitons localized near the nanoslits to the locally enhanced evanescent near field of the SPP.

\section{Coherent ultrafast manipulation of the Rabi energy}

The linear spectra strongly suggest a periodic energy exchange between excitons and SPPs as the physical cause for the clear anti-crossing. Due to the large strength of $\hbar \Omega_{N M S}$, the exchange occurs on an exceedingly fast time scale of $10 \mathrm{fs}$, making its real-time observation
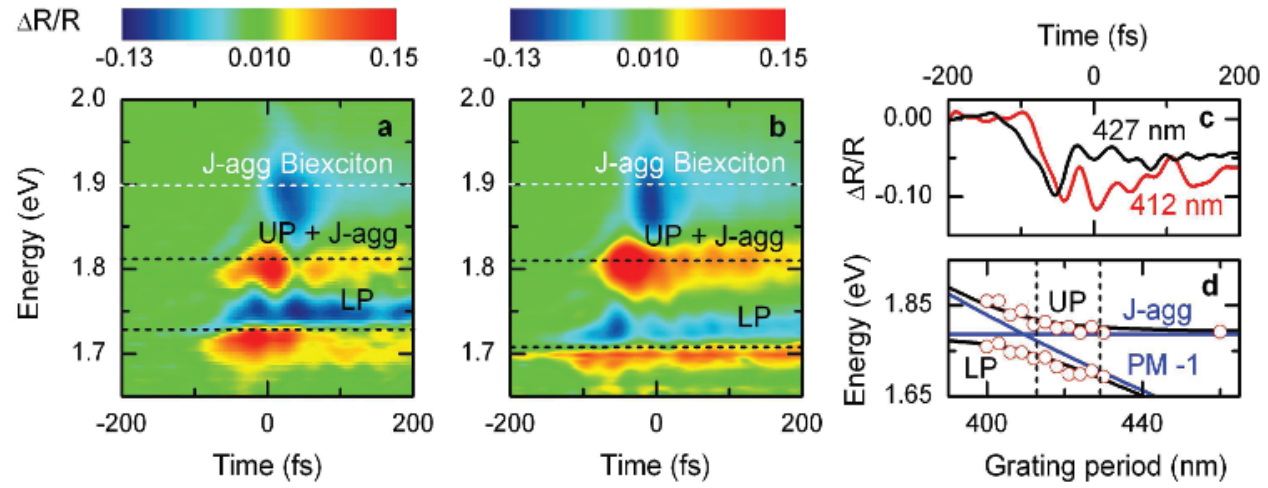

Figure 2: Ultrafast coherent pump-probe spectroscopy of hybrid system depicted in Fig. 1a. An ultrashort sub-15-fs pump laser coherently excites both UP and LP resonances (black dashed lines) and corresponding differential reflectivity changes are probed as a function of the time delay. The measurement in (a) is performed for a grating with a period of $412 \mathrm{~nm}$, exactly at the expected crossing of the exciton and SPP dispersion relations. In (b), a finite detuning is introduced by changing the grating period to $427 \mathrm{~nm}$. (c) Evolution of $\Delta \mathrm{R} / \mathrm{R}$ at LP resonances, $1.76 \mathrm{eV}(412 \mathrm{~nm})$ and $1.73 \mathrm{eV}(427 \mathrm{~nm})$ showing ultrafast Rabi oscillations and (d) Dispersion relation deduced from the assignment of the resonances in pump-probe experiments on samples with different grating periods.

challenging. We have performed angle-resolved pump-probe spectroscopy on the hybrid system depicted in Fig. 1a using sub-15 fs pulses, sufficient to temporally resolve coherent exciton-SPP wavepacket dynamics. Figure 2 shows representative data displaying differential reflectivity spectra 
recorded on two different gratings with periods of (a) $412 \mathrm{~nm}$ (zero exciton-SPP detuning) and (b) $427 \mathrm{~nm}$ (finite detuning), respectively. Experiments on different samples (d) confirm the polariton resonance assignment.

To analyze these results and to uncover the underlying physics, we have performed extensive density matrix simulations of the coupled system considering the molecular aggregate as a three level system exhibiting an exciton-biexciton transition while the SPP mode is modeled as a photonlike oscillator [2,3]. Essentially, the following scenario emerges. The broadband impulse excitation couples to both the UP and LP modes and launches a plasmon-like wavepacket. This initiates a coherent radiative transfer of energy between SPPs and excitons and induces population pulsations between the two systems. The exciton population (with probability $n_{x}(t)$ ) bleaches the exciton absorption and thus transiently reduces the normal mode splitting $\hbar \Omega_{N M S}(t) \propto \sqrt{1-2 n_{x}(t)}$. The material polarization therefore averages over different time-varying normal mode splittings and this gives rise to a transient blue shift of the lower-frequency LP resonance and hence to a dispersive LP nonlinearity as seen in Fig. 2. The magnitude of this shift is given by the time-average $\left\langle\Omega_{N M S}(t)\right\rangle$ of the splitting over the lifetime of the probe-induced polariton wavepacket. This magnitude - and thus the amplitude of $\Delta R$ - is large whenever pump and probe pulses create in-phase wavepackets at $\tau=n T_{R}$, i.e., when the two wavepackets are preferentially localized in the same quantum system. The differential reflectivity change of the LP resonance in Fig. 2 therefore directly probes the dynamics of exciton-SPP Rabi oscillations in real time. Increasing the detuning alters the exciton/SPP fraction in the mixed polariton wavefunctions and hence reduces the contrast of the modulation (Fig. 2b).

\section{Conclusion}

Our experiments demonstrate that induced coherent wavepacket motion gives rise to an ultrafast periodic modulation of the coupling energy and the polariton resonance positions due to the periodic change in exciton density. The all-optical coherent manipulation occurring on a 10 -fs timescale, is fully reversible and can be considered as a new approach to control the hybrid nanostructure response. We anticipate that our results will be relevant for the development of novel technologically important active plasmonic applications.

\section{References}

1. T. Schwartz, J. A. Hutchison, C. Genet, and T. W. Ebbesen, Phys. Rev. Lett. 106, 196405 (2011)

2. P. Vasa, R. Pomraenke, G. Cirmi, E. De Re, W. Wang, S. Schwieger, D. Leipold, E. Runge, G. Cerullo and C. Lienau, ACS Nano 4, 7559 (2010)

3. N. T. Fofang, K. G. Nathaniel, F. Zhiyuan, A. O. Govorov, and N. J. Halas, Nano Lett. 11, 1556 (2011)

4. R. F. Oulton, V. J. Sorger, T. Zentgraf, R-M Ma, C. Gladden, L. Dai, G. Bartal and X. Zhang, Nature, 461, 629 (2009) 